\title{
High-soluble CGA levels are associated with poor survival in bladder cancer
}

\author{
T Szarvas ${ }^{1,2}$, B Jardin-Watelet ${ }^{3}$, N Bourgoin ${ }^{3}$, M J Hoffmann4, P Nyirády², C Oláh², T Széll², A Csizmarik², \\ B Hadaschik ${ }^{1}$ and H Reis $^{5}$ \\ 'Department of Urology, University Hospital Essen, University of Duisburg-Essen, Essen, Germany \\ 2Department of Urology, Semmelweis University, Budapest, Hungary \\ ${ }^{3}$ Thermo Fisher Scientific Cezanne SAS, Clinical Diagnostics Division, Nimes, France \\ ${ }^{4}$ Department of Urology, Medical Faculty, Heinrich-Heine-University Duesseldorf, Duesseldorf, Germany \\ ${ }^{5}$ Institute of Pathology, University Hospital Essen, University of Duisburg-Essen, Essen, Germany
}

Correspondence should be addressed to T Szarvas: sztibusz@gmail.com

\begin{abstract}
Recently, a neuroendocrine-like molecular subtype has been discovered in muscleinvasive urothelial bladder cancer (BC). Chromogranin A (CGA) is a widely used tissue and serum marker in neuroendocrine tumors. Our aim was to evaluate serum CGA (sCGA) concentrations and their associations with clinical and follow-up data in $\mathrm{BC}$ and renal cell carcinoma (RCC). sCGA concentrations were analyzed in the following cohorts: (1) BC training set $(n=188)$, (2) BC validation set $(n=125),(3)$ RCC patients $(n=77),(4)$ healthy controls $(n=97)$. CGA immunohistochemistry and RT-qPCR analyses were performed in 20 selected FFPE and 29 frozen BC tissue samples. Acquired data were correlated with clinicopathological parameters including comorbidities with known effect on SCGA as well as with patients' follow-up data. sCGA levels were significantly higher in BC but not in RCC patients compared to healthy controls. High sCGA levels were independently associated with poor overall and disease-specific survival both in the $\mathrm{BC}$ training $(P<0.001$, $P=0.002)$ and validation set $(P=0.009, P=0.017)$. SCGA levels were inversely correlated with glomerulus filtrating rate (GFR) and linearly correlated with creatinine clearance and urea concentrations. These correlations were not related to the prognostic value of sCGA. Tissue CGA levels were low to absent independently of sCGA concentrations. Our results demonstrate elevated levels and an independent prognostic value for SCGA in BC but not in RCC. Despite the significant correlation between SCGA and GFR, the prognostic relevance of sCGA seems not related to impaired renal function or other comorbidities.
\end{abstract}

\section{Key Words}

- bladder cancer

- chromogranin A

- CGA

- neuroendocrine

- serum

\section{Introduction}

Chromogranin A (CGA) is a $49 \mathrm{kDa}$ glycoprotein encoded by the chromogranin A gene on chromosome 14q32.12. It belongs to the granin family of secretory proteins that are ubiquitous in the nervous, endocrine and immune systems. It is predominantly expressed in neuroendocrine cells and plays a role in the biogenesis of secretory granules (1). CGA may be secreted into the blood as a full-length protein or fragments after cleavage. While definite functions of CGA and its peptides have not been fully elucidated, they may be involved in the formation of dense-core granules, catecholamine and parathyroid hormone secretion, lipid metabolism, immune properties and reproduction (2). Furthermore, CGA peptides regulate a number of cellular processes including cell proliferation and angiogenesis (3).

When a tumor develops in a neuroendocrine tissue, it becomes the main source of circulating CGA (4). CGA became therefore a routinely available however debated

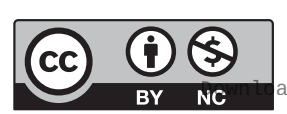


marker for the diagnosis and monitoring of neuroendocrine tumors (5). In addition to neuroendocrine tumors, neuroendocrine activity and elevated CGA serum levels can also be detected in other tumors that are not derived from neuroendocrine tissues such as breast, prostate and ovarian cancer $(6,7,8)$. Recent comprehensive transcriptomic analysis revealed a distinct molecular subtype of urothelial bladder cancer (BC), which can be characterized by neuroendocrine-like expression profile. One of the typically overexpressed genes in this molecular subtype is CGA (9). To the best of our knowledge, serum levels of CGA in urothelial cancer of the urinary bladder and in renal cell cancer (RCC) have not been evaluated yet. However, urinary CGA levels in $44 \mathrm{BC}$ patients were found to be homogenously low (10).

In addition to malignant diseases, increased CGA serum concentrations have been found in association with some non-malignant diseases, such as heart disease, acute coronary syndrome $(11,12,13)$, endometriosis, leiomyoma (14), ulcerative colitis and Chron's disease (15). As some CGA fragments are cleared by hepatic metabolism and renal excretion, major hepatic failure such as chronic hepatitis and cirrhosis or even modest renal failure can lead to increased serum CGA levels $(13,16,17,18)$.

Recently, we assessed the prognostic value of soluble CGA (sCGA) in clinically localized prostate cancer (PCA) (19). Performing these analyses we measured a number of serum samples from BC patients and detected surprisingly high sCGA values in some cases. This incidental observation led us to measure sCGA levels in a large number of patients with BC. In a subsequent analysis we extended the measurement of sCGA to an independent cohort of $\mathrm{BC}$ patients and assessed preoperative sCGA levels also in serum samples of RCC patients. In addition, we analyzed tissue CGA expression both at the gene expression and protein levels. Acquired data were compared to clinicopathological and follow-up data as well as to comorbidities of patients.

\section{Materials and methods}

\section{Clinical samples}

A total number of 390 patients from three patient cohorts and 97 age-matched healthy individuals were included in this study. The first BC cohort (training set) included 188 urothelial BC patients, the second BC cohort (validation set) included 125 urothelial $\mathrm{BC}$ patients while the third patient cohort consisted of 77 RCC (66x clear cell RCC, $6 \times$ chromophobe RCC and $5 \times$ papillary RCC) patients.
Patients in the training and validation were treated at the same university hospital at a different time period. For the training $\mathrm{BC}$ cohort serum samples, while for the validation cohort plasma samples were available for analysis. All samples were collected preoperatively in a single academic center. None of the patients received neoadjuvant chemotherapy prior to surgery. Clinical data including age, gender, tumor stage, grade, lymph node (LN) status, presence of distant metastasis and details on survival were obtained from the medical records and relevant offices. Overall survival (OS) and diseasespecific survival (DSS) were recorded as time from sample collection to the relevant event or censoring. Parameters of renal function including GFR (glomerular filtration rate), creatinine and urea concentrations were available for the training cohort, while comorbidities were noted for all patients. Patients' characteristics for these cohorts are given in Table 1 . The study was performed according to the Declaration of Helsinki and the institutional ethics committee (Ethik-Kommission der Medizinischen Fakultät der Universität Duisburg-Essen) approved the study protocol. The ethical permission number is 14-5808BO. All patients signed consent to an institutional review board-approved protocol before sample collection.

Blood samples were collected into EDTA-coated Monovette tubes for plasma samples and into serum (S)-Monovette (Sarstedt, Nümbrecht, Germany) for serum samples and were stored at $-80^{\circ} \mathrm{C}$ until analyzed.

In addition to blood samples corresponding formalinfixed and paraffin-embedded (FFPE) tumor tissues from 20 $\mathrm{BC}$ patients as well as 29 frozen tumor tissue samples were analyzed by immunohistochemistry and RT-qPCR analysis.

\section{SCGA analysis by the KRYPTOR method}

CGA levels were measured on the fully automated B.R.A.H.M.S KRYPTOR instrument (Thermo Scientific B.R.A.H.M.S GmbH, Hennigsdorf/Berlin, Germany) using the B.R.A.H.M.S. CGA II homogeneous sandwich fluoroimmuno-assay as described previously $(20,21,22)$. The functional sensitivity of this assay is $13.7 \mathrm{ng} / \mathrm{mL}$. Two commercial CGA controls were measured in each assay. The quality controls mean intra-assay variabilities for duplicate measurements were 2.7 and $1.4 \%$, while the inter-assay variabilities were 4.7 and $3.4 \%$.

\section{Immunohistochemical analysis}

Tissue CGA levels were immunohistochemically analyzed in FFPE tumor sections from ten $\mathrm{BC}$ patients with the highest

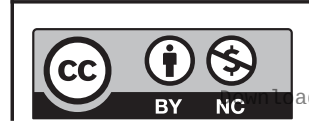

This work is licensed under a Creative Commons Attribution-NonCommercial 4.0 International License. ded from Bioscientifica.com at 04/26/2023 10:23:50AM 
Table 1 Patients' and follow-up characteristics.

\begin{tabular}{lc}
\hline Variables/cohorts & BCA training set \\
\hline Total number of patients & 188 \\
Sample & Serum \\
Age, median (range) & $71(21-90)$ \\
Gender & 149 \\
$\quad$ Male & 39 \\
Female & \\
LN or distant metastasis & 156 \\
N0/M0 & 32 \\
N+/M+ & \\
Stage & 8 \\
Cis & 81 \\
pTa & 19 \\
pT1 & 28 \\
pT2 & 27 \\
pT3 & 25 \\
pT4 & \\
Grade & 37 \\
G1 & 93 \\
G2 & 58 \\
G3 & 63.9 \\
CGA level median (ng/mL) & $14.6-1785.5$ \\
CGA level range (ng/mL) & 56 \\
Number of patients died & 39 \\
Disease-specific deaths & $24(1-71)$ \\
Follow-up, months, median (range) & \\
\hline
\end{tabular}

\begin{tabular}{c}
\hline BCA validation set \\
\hline 125 \\
Plasma \\
$65(36-89)$ \\
100 \\
25 \\
106 \\
19 \\
5 \\
25 \\
21 \\
36 \\
26 \\
12 \\
19 \\
37 \\
69 \\
30.9 \\
$1.0-400.9$ \\
78 \\
56 \\
$29(1-156)$ \\
\hline
\end{tabular}

\begin{tabular}{c}
\hline RCC \\
\hline 77 \\
Serum \\
$64(32-87)$ \\
53 \\
24 \\
68 \\
9
\end{tabular}

\begin{tabular}{c}
\hline Controls \\
\hline 97 \\
Serum \\
$63(52-79)$
\end{tabular}

BCA, bladder cancer; LN, lymph node; RCC, renal cell cancer.

and ten patients with the lowest sCGA levels. From one tumor containing block in each case, $2 \mu \mathrm{m}$ thick sections were cut and automated immunohistochemistry (CGA: Leica, Clone: 5H7, dilution: 1:100, incubation: $24 \mathrm{~min}$, $37^{\circ} \mathrm{C}$, pre-treatment: $\mathrm{CC} 1,40 \mathrm{~min}$ ) was performed using the Benchmark Ultra System (Ventana Systems, Tucson, AZ, USA) according to manufacturer's instructions. Any detectable typical granular CGA immunoreactivity was evaluated as positive and the percentage of positive tumor cells was semiquantitatively evaluated. In every case, the whole tumor was taken into account for estimation of percentage of positive cells. In addition we evaluated stroma cells for potential CGA staining which was absent in every case.

\section{Gene expression analysis}

CGA gene expression levels were analyzed in 29 frozen BC tissue samples for which corresponding circulating CGA concentrations were available. Only biopsies containing $\geq 70 \%$ tumor cells were selected for RNA isolation and cDNA synthesis which were performed as described earlier (23). Quantitative real-time PCR was performed on a Lightycler (Roche) using QuantiTect SYBR Green. Concentration values are calculated based on standard curves carried out for each gene and each run. TBP (forward: ACAACAGCCTGCCACCTTA, reverse: GAATAGGCTGTGGGGTCAGT) and SDHA (forward: GCCAGGACCTAGAGTTTGTTCA, reverse: GAAT AGGCTGTGGGGTCAGT) were measured as reference genes (24) and a normalization factor was calculated for each sample using their geometric mean (25). Expression values of CGA (forward: CTCCAGGTCCGAGGCTAC, reverse: GACAGGCTCTCCAGCTCC) are given relative to this normalization factor.

\section{Statistical analysis}

For paired group comparisons, the nonparametric, twosided Wilcoxon rank-sum test (Mann-Whitney test) was applied. Univariate OS and DSS analyses were done using Kaplan-Meier log-rank test and univariate Cox analysis. For multiple analyses, the Cox proportional hazards regression model was used. Parameters which were associated with patients' survival $(P \leq 0.150)$ were included in the multivariable analyses. In all tests, $P$ values $<0.05$ were considered statistically significant. Correlation between CGA and GFR/creatinine concentration/urea concentration were examined by using Spearman's correlation coefficients. All statistical analyses were done with the SPSS software package (24.0; SPSS). 


\section{Results}

\section{Clinical characteristics of patients}

For the training cohort of 188 BC patients, 56 patients died during the follow-up period, 39 of them BC related. In 32 patients, metastasis was detected at diagnosis (26× LN, $2 \times$ distant and $4 \times \mathrm{LN}$ and distant metastases) (Table 1 ). The median values for the GFR, creatinine and urea were $62 \mathrm{~mL} / \mathrm{min} / 1.73 \mathrm{~m}^{2}$ (range: $24-105$ ), $1.13 \mathrm{mg} / \mathrm{dL}$ (range: $0.69-2.79$ ) and $16.0 \mathrm{mg} / \mathrm{dL}$ (range: 6-50), respectively. Chronic heart disease, angina and diabetes mellitus were present in 102, 3 and 36 of 188 patients, respectively.

For the validation cohort of $125 \mathrm{BC}$ patients, 78 patients died during the follow-up period, 56 of them BC related. Median survival time was 29 months. In 19 patients, metastasis was detected at diagnosis $(15 \times \mathrm{LN}, 1 \times$ distant and $3 \times$ LN and distant metastases) (Table 1). Data on comorbidities were available for 96 of 125 patients. Chronic heart disease and diabetes mellitus was known for 32 and 13 of 96 patients, while no patient with angina was noted.

For the RCC cohort of 77 patients, 42 patients died during the follow-up period (24 RCC related). The median survival time was 110 months. In nine patients metastasis was detected at diagnosis $(2 \times \mathrm{LN}, 5 \times$ distant and $2 \times \mathrm{LN}$ and distant metastases) (Table 1 ). Comorbidity data were available for $72 / 77$ patients. Chronic heart disease, angina and diabetes mellitus were present in 9,2 and 7 of 72 RCC patients.

\section{sCGA levels in controls}

Serum CGA levels were measured in 97 healthy controls (Table 1). We found no difference in CGA levels between control males and females. In contrast, serum CGA levels were significantly higher in elderly controls in both control groups $(P<0.001)$. In five cases both plasma and corresponding serum samples were available for analysis. Serum CGA levels (average: $44.6 \mathrm{ng} / \mathrm{mL}$ range: $18.2-72.7 \mathrm{ng} / \mathrm{mL}$ ) were consequently higher compared to plasma (average: $33.0 \mathrm{ng} / \mathrm{mL}$ range: $13.7-56.1 \mathrm{ng} / \mathrm{mL}$ ).

\section{sCGA levels in BC patients}

CGA levels were significantly higher in serum samples of the training $\mathrm{BC}$ cohort compared to plasma samples of the validation cohort $(P<0.001)$. sCGA levels were significantly higher in $\mathrm{BC}$ patients compared to age-matched controls $(P<0.001)$ (Fig. 1). Furthermore, similar to the findings in the control group, sCGA levels were significantly higher in elderly subjects both in the $\mathrm{BC}$ training and validation cohorts $(P=0.026, P=0.001)$. We found no significantly different sCGA levels between metastatic vs non-metastatic or between high-stage and low-stage BCs. We observed higher sCGA levels in men in the training cohort; however, this correlation could not be confirmed in the validation cohort $(P=0.009, P=0.218)$. CGA serum levels were higher in patients with low-grade tumors; however, this was not confirmed in the plasma samples of the validation cohort (Table 2). Overall sCGA levels of BC patients were higher than those of controls, RCC patients or patients with local or progressed stages of prostate cancer (Fig. 1) (19).

\section{sCGA levels in renal cell carcinoma}

We found no difference in sCGA levels between RCC patients and age- and gender-matched controls. Serum CGA levels were higher in female patients. No such correlation was observed in the control group. Circulating CGA levels showed no correlation with tumor stage, grade, histological subtype and the presence of LN or distant metastases (Fig. 1 and Table 2).

\section{Univariable and multivariable survival analysis}

Both in the training and validation $\mathrm{BC}$ cohorts tumor stage, grade and the presence of metastasis were significantly associated with patients' OS and DSS.

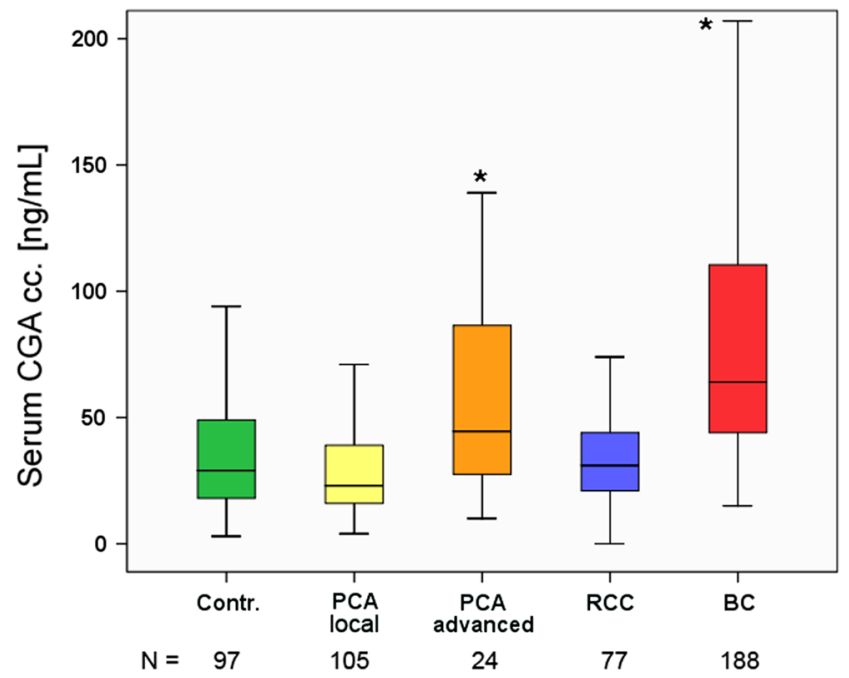

\section{Figure 1}

Serum CGA concentrations in RCC, BCA and in PCA (PCA data are generated in a formerly published study by using the same assay (19). CGA serum levels are elevated in advanced PCA and in BC while they are unchanged in RCC and local prostate cancer PCA. *Significant difference compared to controls. 

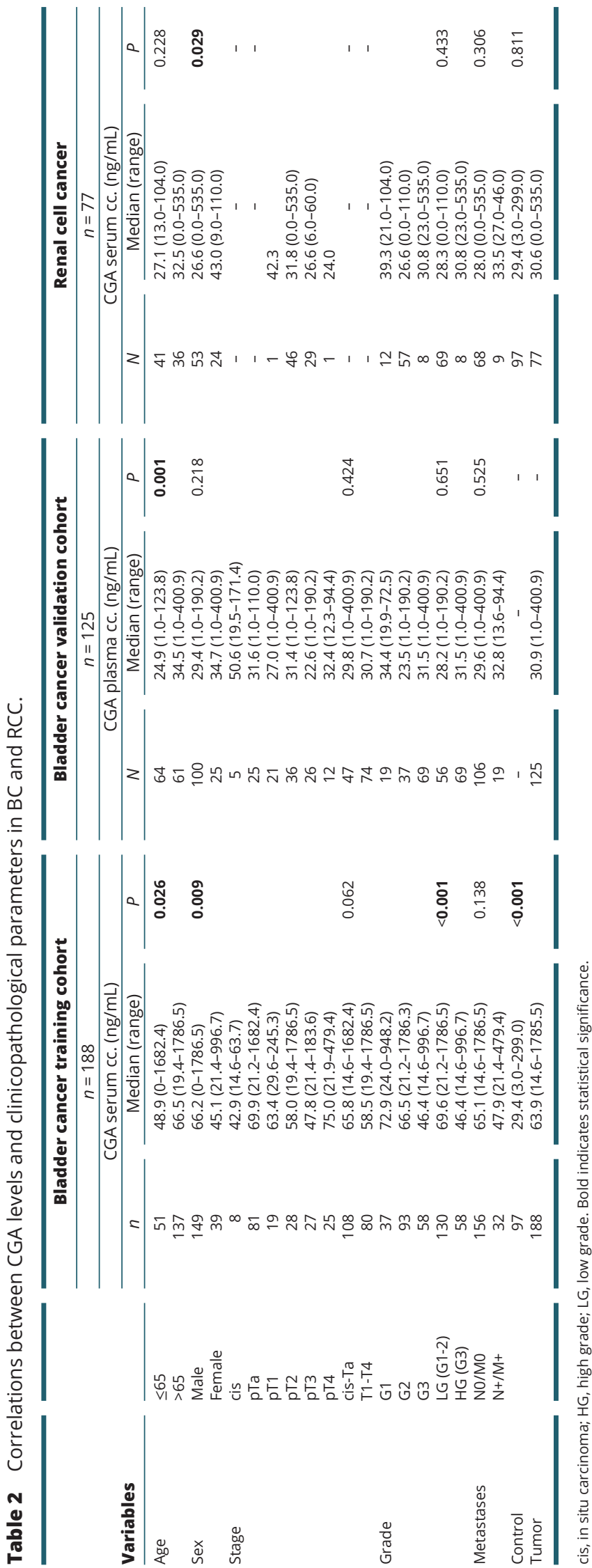

https://ec.bioscientifica.com https://doi.org/10.1530/EC-19-0068

(c) 2019 The authors Published by Bioscientifica Ltd
High serum CGA levels were significantly associated with poor OS and DSS $(P=0.002, P=0.026)$, while plasma CGA levels (validation set) were correlated with OS $(P=0.025)$ and tended to correlate with DSS $(P=0.150)$ (Fig. 2 and Table 3). In multivariable analyses, presence of metastasis and high CGA levels were independently associated with poor OS and DSS in both training and validation cohorts (Table 3). In the subgroup of patients who underwent radical cystectomy, sCGA proved to have an independent prognostic factor for OS and DSS in both BC cohorts (Fig. 2 and Supplementary Table 1, see section on supplementary data given at the end of this article).

In RCC patients, the presence of LN or distant metastases were associated with poor OS and DSS $(P=0.038, \quad P=0.006)$ (Supplementary Table 2). Tumor stage was associated with DSS $(P=0.010)$, while tumor grade correlated with OS $(P=0.003)$. Circulating CGA concentrations showed no correlation with patients OS or DSS.

\section{Tissue CGA levels in BC}

CGA gene expression levels were determined in 29 BC tissues. Overall, CGA mRNA expression was low $(n=14)$ to undetectable $(n=15)$ in almost all samples. The Ct values were higher than 30 except in one tumor sample, while in the same samples housekeeping gene expression was robust with median Cq values of 25.23 for TBP and 22.94 for SDHA (Supplementary Table 3). No correlation could be observed between soluble CGA and tissue CGA mRNA expression levels.

CGA immunohistochemistry was done in 20 selected FFPE BC tissues, resulting in negative staining in the majority of cases $(n=16)$. Three of the patients with high sCGA concentration $(n=10)$ showed CGA immunopositivity in $1-3 \%$ of tumor cells, while in the sCGA low group 1 sample showed CGA-positive immunostaining in $1 \%$ of tumor cells.

\section{sCGA levels and comorbidities}

We retrieved relevant comorbidities including chronic heart disease $(n=102)$, angina $(n=3)$, diabetes mellitus $(n=36)$ and reduced renal function (GFR $<60 \mathrm{~mL} / \mathrm{min}$, $n=76)$ for the training cohort $(n=188)$ to assess their effect on CGA levels in our BC patients. We found no significantly different sCGA values in patients with and without chronic heart disease, diabetes mellitus or angina. In contrast, the median sCGA concentrations in patients with reduced renal function (defined as GFR $<60 \mathrm{~mL} / \mathrm{min} / 1.73 \mathrm{~m}^{2}$ ) 

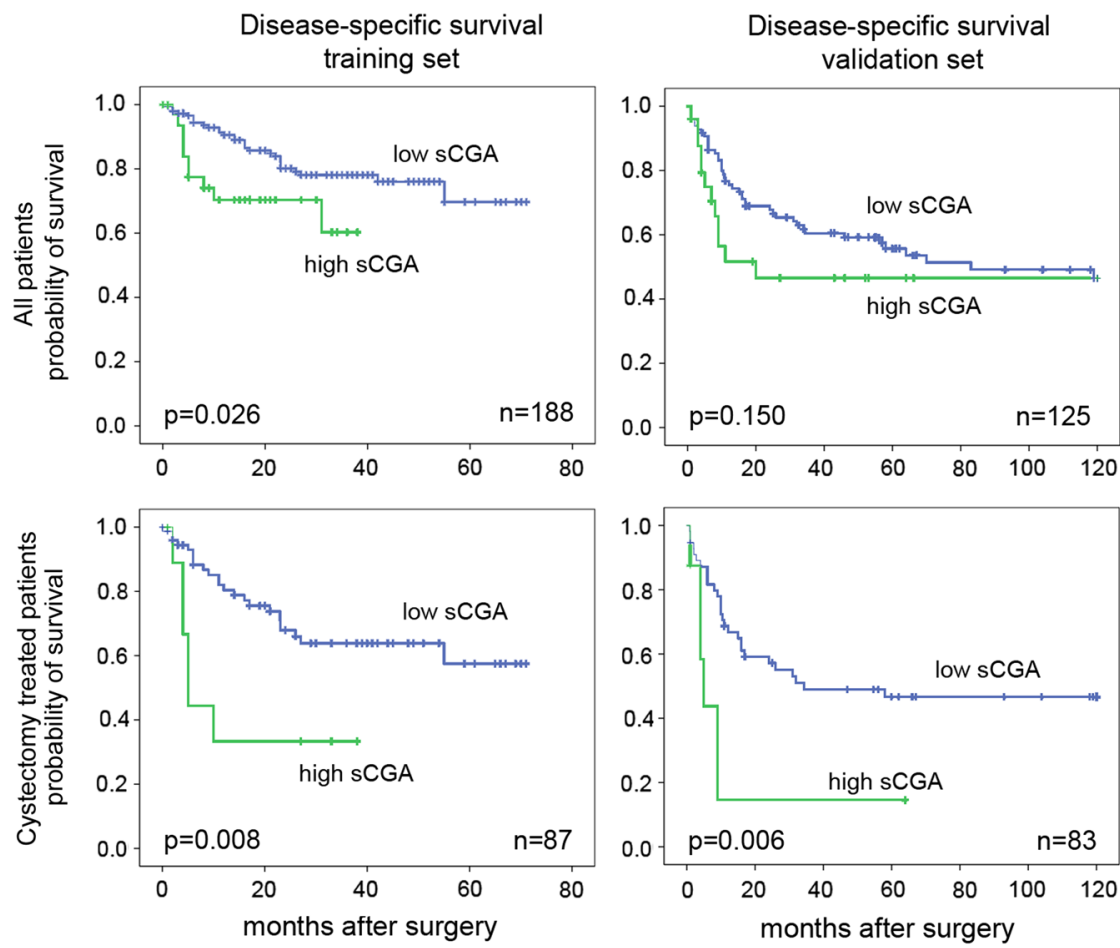

Figure 2

Kaplan-Meier disease-specific survival curves stratified by sCGA levels in training (left) and validation (right) cohorts. Survival analyses were performed in all patients (upper panels) and in the subgroups of patients who were treated by radical cystectomy (lower panels).

was significantly higher (median 83.3) than in those with normal renal function (median 51.3). Circulating CGA levels were inversely correlated with GFR values (correlation coefficient: $-0.456, P<0.001)$. None of the considered comorbidities had any significant effect on OS or DSS. Only reduced renal function showed a trend toward an impact on OS $(P=0.066)$ but not on DSS $(P=0.334)$ survival in the subgroup of patients with non-muscle-invasive $\mathrm{BC}$ (MIBC).

CGA levels were associated with poor OS independent of GFR as high CGA serum concentrations correlated with poor OS in patients with normal renal function $(P=0.008)$ and tended to correlate with poor OS in patients with reduced renal function $(P=0.089)$.

\section{Discussion}

In the present study, analyzing circulating sCGA levels in BC and RCC, for the first time, we found significantly elevated sCGA levels in BC compared to controls and high sCGA levels were independently associated with poor OS and DSS. These correlations could be confirmed in an independent cohort of BC patients. In contrast, no similar findings could be observed in RCC.

Serum CGA levels are used for diagnostic and monitoring purposes in various neuroendocrine tumors. According to The Human Protein Atlas, non-neoplastic tissues of pancreas and the gastrointestinal tract, especially stomach, duodenum, small intestine and rectum express moderate levels of CGA. Accordingly, elevated sCGA levels were detected in non-neuroendocrine tumors arising from these tissues (26). In addition, also non-neuroendocrine carcinomas of the breast, prostate, ovary and hepatocellular carcinomas may exhibit elevated sCGA levels $(5,26,27)$.

No CGA expression has been observed in any of the main histological subtypes of RCC, while the CGA expression of urothelial BC has not been systematically assessed yet (28). We formerly found surprisingly high sCGA concentration in serum samples of a small subset of BC patients. These results led us to assess the sCGA levels in a large number of BC patients. Our present results demonstrate two-fold significantly elevated sCGA levels in BC patients compared to age-matched controls. We found no significant correlation between tumor stage and sCGA levels. Most importantly, high sCGA levels (upper 20\% of cases) were independently associated with poor OS and DSS. In contrast, sCGA levels in RCC were similar to those of controls and sCGA does not seem to prove any prognostic value in RCC patients. To confirm the unexpected findings in $\mathrm{BC}$, we determined sCGA levels in a validation cohort of $\mathrm{BC}$ patients. Using the same method for setting the cut-off point (upper 20\%), we could confirm the independent prognostic relevance sCGA in BC. As in prostate adenocarcinoma the presence 
Table 3 Cox survival analysis in BC patients.

\begin{tabular}{|c|c|c|c|c|c|c|c|c|c|c|c|c|c|}
\hline \multirow{2}{*}{\multicolumn{2}{|c|}{ Training cohort }} & \multicolumn{6}{|c|}{ Univariable survival analyses } & \multicolumn{6}{|c|}{ Multivariable survival analyses } \\
\hline & & \multicolumn{3}{|c|}{ OS } & \multicolumn{3}{|c|}{ DSS } & \multicolumn{3}{|c|}{ OS } & \multicolumn{3}{|c|}{ DSS } \\
\hline \multicolumn{2}{|l|}{$n=188$} & HR & $95 \% \mathrm{Cl}$ & $P$ & $\mathrm{HR}$ & $95 \% \mathrm{Cl}$ & $P$ & $\mathrm{HR}$ & $95 \% \mathrm{Cl}$ & $P$ & $\mathrm{HR}$ & $95 \% \mathrm{Cl}$ & $P$ \\
\hline \multirow{2}{*}{ Age } & $\leq 65$ years & ref. & & & ref. & & & ref. & & & ref. & & \\
\hline & $>65$ years & 1.727 & $0.892-3.343$ & 0.165 & 1.951 & $0.860-4.427$ & 0.180 & - & - & - & - & - & - \\
\hline \multirow[t]{2}{*}{ Sex } & Female & ref. & & & ref. & & & ref. & & & ref. & & \\
\hline & $\begin{array}{l}\text { Male } \\
\text { Ta }\end{array}$ & $\begin{array}{c}0.660 \\
\text { ref. }\end{array}$ & $0.369-1.179$ & 0.160 & $\begin{array}{c}0.447 \\
\text { ref. }\end{array}$ & $0.247-0.918$ & 0.027 & $\begin{array}{l}0.840 \\
\text { ref. }\end{array}$ & $0.463-1.524$ & 0.566 & $\begin{array}{c}0.655 \\
\text { ref. }\end{array}$ & $0.334-1.284$ & 0.218 \\
\hline Grade & $\begin{array}{l}\text { T1-T4 } \\
\text { LG }\end{array}$ & $\begin{array}{l}3.705 \\
\text { ref. }\end{array}$ & $2.093-6.558$ & $<0.001$ & $\begin{array}{l}5.449 \\
\text { ref. }\end{array}$ & $2.583-11.495$ & $<0.001$ & $\begin{array}{l}1.798 \\
\text { ref. }\end{array}$ & $0.869-3.717$ & 0.114 & $\begin{array}{l}3.057 \\
\text { ref. }\end{array}$ & $1.186-7.882$ & 0.021 \\
\hline Metastases & $\begin{array}{l}\mathrm{HG} \\
\mathrm{NO} / \mathrm{MO}\end{array}$ & $\begin{array}{c}2.489 \\
\text { ref. }\end{array}$ & $1.470-4.212$ & 0.001 & $\begin{array}{c}2.014 \\
\text { ref. }\end{array}$ & $1.547-5.490$ & 0.001 & $\begin{array}{c}1.211 \\
\text { ref. }\end{array}$ & $0.606-2.417$ & 0.588 & $\begin{array}{c}1.052 \\
\text { ref. }\end{array}$ & $0.470-2.354$ & 0.902 \\
\hline sCGA level & $\begin{array}{l}\mathrm{N}+/ \mathrm{M}+ \\
<147 \mathrm{ng} / \mathrm{mL}\end{array}$ & $\begin{array}{l}5.523 \\
\text { ref. }\end{array}$ & $3.213-9.492$ & $<0.001$ & $\begin{array}{l}7.125 \\
\text { ref. }\end{array}$ & $3.757-13.512$ & $<0.001$ & $\begin{array}{l}3.913 \\
\text { ref. }\end{array}$ & $1.929-7.934$ & $<0.001$ & $\begin{array}{c}4.337 \\
\text { ref. }\end{array}$ & $1.919-9.801$ & $<0.001$ \\
\hline cut-off $20 \%$ & $>147 \mathrm{ng} / \mathrm{mL}$ & 2.553 & $1.406-4.566$ & 0.002 & 2.295 & $1.106-4.764$ & 0.026 & 3.304 & $1.791-6.098$ & $<0.001$ & 3.366 & $1.565-7.241$ & 0.002 \\
\hline \multicolumn{2}{|c|}{ Validation cohort } & \multicolumn{3}{|c|}{ OS } & \multicolumn{3}{|c|}{ DSS } & \multicolumn{3}{|c|}{ OS } & \multicolumn{3}{|c|}{ DSS } \\
\hline \multicolumn{2}{|l|}{$n=125$} & $\mathrm{HR}$ & $95 \% \mathrm{Cl}$ & $P$ & $\mathrm{HR}$ & $95 \% \mathrm{Cl}$ & $P$ & $\mathrm{HR}$ & $95 \% \mathrm{Cl}$ & $P$ & $\mathrm{HR}$ & $95 \% \mathrm{Cl}$ & $P$ \\
\hline \multirow[t]{2}{*}{ Age } & $\leq 65$ years & ref. & & & ref. & & & ref. & & & ref. & & \\
\hline & $\begin{array}{l}>65 \text { years } \\
\text { Female }\end{array}$ & $\begin{array}{c}1.760 \\
\text { ref. }\end{array}$ & $1.105-2.804$ & 0.167 & $\begin{array}{c}1.687 \\
\text { ref. }\end{array}$ & $0.984-2.893$ & 0.257 & $\begin{array}{c}- \\
\text { ref. }\end{array}$ & - & - & $\begin{array}{c}- \\
\text { ref. }\end{array}$ & - & - \\
\hline Stage & $\begin{array}{l}\text { Male } \\
\text { Ta }\end{array}$ & $\begin{array}{c}0.651 \\
\text { ref. }\end{array}$ & $0.383-1.108$ & 0.114 & $\begin{array}{c}0.748 \\
\text { ref. }\end{array}$ & $0.394-1.420$ & 0.374 & $\begin{array}{c}0.611 \\
\text { ref. }\end{array}$ & $0.344-1.087$ & 0.094 & ref. & - & - \\
\hline \multirow[t]{2}{*}{ Grade } & $\begin{array}{l}\text { T1-T4 } \\
\text { LG (G1-G2) }\end{array}$ & $\begin{array}{c}2.070 \\
\text { ref. }\end{array}$ & $1.227-3.492$ & 0.006 & $\begin{array}{c}2.301 \\
\text { ref. }\end{array}$ & $1.243-4.259$ & 0.008 & $\begin{array}{c}1.972 \\
\text { ref. }\end{array}$ & $0.993-3.915$ & 0.052 & $\begin{array}{c}2.920 \\
\text { ref. }\end{array}$ & $1.197-7.123$ & 0.019 \\
\hline & $\begin{array}{l}\text { HG (G3) } \\
\text { NO/MO }\end{array}$ & $\begin{array}{l}1.795 \\
\text { ref. }\end{array}$ & $1.116-2.888$ & 0.016 & $\begin{array}{l}1.787 \\
\text { ref. }\end{array}$ & $1.030-3.103$ & 0.039 & $\begin{array}{l}1.106 \\
\text { ref. }\end{array}$ & $0.641-1.910$ & 0.717 & $\begin{array}{c}0.937 \\
\text { ref. }\end{array}$ & $0.496-1.769$ & 0.840 \\
\hline sCGA level & $\begin{array}{l}\mathrm{N}+/ \mathrm{M}+ \\
<51 \mathrm{ng} / \mathrm{mL}\end{array}$ & $\begin{array}{c}3.285 \\
\text { ref. }\end{array}$ & $1.857-5.813$ & $<0.001$ & $\begin{array}{l}4.409 \\
\text { ref. }\end{array}$ & $2.388-8.141$ & $<0.001$ & $\begin{array}{l}3.120 \\
\text { ref. }\end{array}$ & $1.641-5.932$ & 0.001 & $\begin{array}{l}3.715 \\
\text { ref. }\end{array}$ & $1.895-7.284$ & $<0.001$ \\
\hline cut-off $20 \%$ & $>51 \mathrm{ng} / \mathrm{mL}$ & 1.851 & $1.082-3.166$ & 0.025 & 1.596 & $0.837-3.043$ & 0.150 & 2.161 & $1.207-3.866$ & 0.009 & 2.297 & $1.162-4.543$ & 0.017 \\
\hline
\end{tabular}

Bold indicates statistical significance.

and rate of neuroendocrine tumor cells is suggested to be associated with more aggressive behavior of the tumor, we formerly assessed the sCGA levels in clinically localized and progressed stages of PCA (19). Interestingly, comparing the sCGA levels between PCA and $\mathrm{BC}$, we observed significantly higher concentrations in urothelial BC.

Radical cystectomy is the standard treatment for muscleinvasive BC (MIBC). However, this 'gold standard' only provides 5 -year survival in about $50 \%$ of patients, showing that MIBC represents a prognostically heterogeneous group of patients (29). To date there are no routinely used markers to identify patients most likely to benefit from radical cystectomy alone and those who need additional treatments. Therefore, there is a clear need for novel prognostic biomarkers to ensure adequate risk stratification in MIBC. To address the question of whether sCGA is prognostic in the subset of patients with MIBC who underwent radical surgery, we performed OS and DSS analyses in this subgroup in both the training and the validation set of our BC patients. In both subgroup analyses, high sCGA proved to be an independent predictor of poor OS and DSS suggesting that sCGA may help to preoperatively identify patients who need a more aggressive therapy.
As in prostate cancer, presence of CGA-positive tumor cells were found to be correlated with higher sCGA levels, we hypothesized that high serum CGA levels in BC may originate from CGA-positive neuroendocrine-like tumor cells. This aspect is important as recent TCGA (The Cancer Genome Atlas) data revealed a 'neuroendocrine-like' molecular subtype of MIBCs. Tumors with this subtype show no neuroendocrine histopathological features but can be characterized at the molecular level by high expression of genes physiologically expressed in neuronal tissues such as TUBB2B, GNG4, ENO2, NCAM1, PEG10, PLEKHG4B, SCG2 and CGA (9). As neuroendocrine-like urothelial carcinoma has just recently been described based on gene expression data, no recommendation exists for the immunohistochemical characterization of this molecular subtype. Notably, this subtype has the most devastating prognosis with currently no specific therapeutic recommendation. Our analysis at the protein level identified CGA-positive tumor cells in 3 of 10 BC patients with high sCGA, compared to 1 of 10 CGApositive tissue samples in patients with low sCGA levels. Similarly, low CGA expressions were found at the mRNA level independent of sCGA concentration. Based on these results, we could not confirm that the elevated serum 


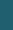

CGA levels in BC originate directly from the tumor cells. Tumor heterogeneity and focal CGA expression may explain the lack of correlation between soluble and tissue CGA expression values. Therefore, the possible link between this neuroendocrine-like molecular BC subtype and elevated sCGA levels should be further analyzed.

Another possible explanation for the elevated sCGA levels might be the presence of comorbidities affecting CGA levels. Decreased renal function, chronic heart failure, angina and diabetes were reported to be associated with elevated sCGA levels. In our study, none of the assessed comorbidities had a significant impact on patients' OS or DSS. Only low GFR values ( $<60 \mathrm{~mL} / \mathrm{min}$ ) in the subgroup of patients with non-muscle-invasive $\mathrm{BC}$ tended to associate with poor OS. This is in accordance with the observation of Rausch et al. who demonstrated that low GFR values are associated with poor prognosis in non-muscle-invasive BC (30). Our data suggest that the prognostic value of sCGA is independent of its correlation with decreased renal function as sCGA levels proved to be prognostic in patients both with normal and decreased renal function.

Our study is limited as serum and plasma sCGA concentrations could not be directly compared. Therefore, the cut-off value used for the stratification of the training set could not be confirmed by analyzing the validation cohort. To overcome this limitation, we used the same principle (upper 20\% percent of the given cohort) to set the cut-off value. Because of these limitations, the prospective evaluation of sCGA levels in BC patients and controls would be necessary to validate our results.

In conclusion, sCGA levels may be implemented in preoperative risk stratification of BC patients. This may help to optimize therapy decisions, especially in patients with MIBC. Our data should be confirmed in a prospective study which may also help to determine the optimal cut-off value for sCGA. In addition, further research should clarify the source of elevated sCGA levels in $\mathrm{BC}$ and a possible correlation with the newly identified molecular subtypes.

\section{Supplementary data}

This is linked to the online version of the paper at https://doi.org/10.1530/ EC-19-0068.

\section{Declaration of interest}

T Szarvas, B Jardin-Watelet and N Bourgoin have patents regarding the method. The other authors have nothing to disclose.

\section{Funding}

The authors would like to thank Prof. Wolfgang Schulz for constructive criticism of the manuscript. This work was supported by NKFIH/FK 124431, NVKP_16-1-2016-004 and the ÙNKP-18-4-SE-66 New National Excellence Program of the Ministry of Human Capacities. T Szarvas was supported by a János Bolyai Research Scholarship of the Hungarian Academy of Sciences.

\section{References}

1 Modlin IM, Gustafsson BI, Moss SF, Pavel M, Tsolakis AV \& Kidd M. Chromogranin A - biological function and clinical utility in neuro endocrine tumor disease. Annals of Surgical Oncology 201017 2427-2443. (https://doi.org/10.1245/s10434-010-1006-3)

2 Lawrence B, Gustafsson BI, Kidd M, Pavel M, Svejda B \& Modlin IM. The clinical relevance of chromogranin A as a biomarker for gastroenteropancreatic neuroendocrine tumors. Endocrinology and Metabolism Clinics of North America 201140 111-134, viii. (https:// doi.org/10.1016/j.ecl.2010.12.001)

3 Kanakis G \& Kaltsas G. Biochemical markers for gastroenteropancreatic neuroendocrine tumours (GEP-NETs). Best Practice and Research: Clinical Gastroenterology 201226 791-802. (https://doi.org/10.1016/j.bpg.2012.12.006)

4 O'Connor DT \& Deftos LJ. Secretion of chromogranin A by peptide-producing endocrine neoplasms. New England Journal of Medicine 1986314 1145-1151. (https://doi.org/10.1056/ NEJM198605013141803)

5 Kidd M, Bodei L \& Modlin IM. Chromogranin A: any relevance in neuroendocrine tumors? Current Opinion in Endocrinology, Diabetes, and Obesity 201623 28-37. (https://doi.org/10.1097/ MED.0000000000000215)

6 Gkolfinopoulos S, Tsapakidis K, Papadimitriou K, Papamichael D \& Kountourakis P. Chromogranin A as a valid marker in oncology: clinical application or false hopes? World Journal of Methodology 2017 7 9-15. (https://doi.org/10.5662/wjm.v7.i1.9)

7 Burgio SL, Conteduca V, Menna C, Carretta E, Rossi L, Bianchi E, Kopf B, Fabbri F, Amadori D \& De Giorgi U. Chromogranin A predicts outcome in prostate cancer patients treated with abiraterone. Endocrine-Related Cancer 201421 487-493. (https://doi.org/10.1530/ ERC-14-0071)

8 Conteduca V, Burgio SL, Menna C, Carretta E, Rossi L, Bianchi E, Masini C, Amadori D \& De Giorgi U. Chromogranin A is a potential prognostic marker in prostate cancer patients treated with enzalutamide. Prostate 201474 1691-1696. (https://doi.org/10.1002/ pros.22890)

9 Robertson AG, Kim J, Al-Ahmadie H, Bellmunt J, Guo G, Cherniack AD, Hinoue T, Laird PW, Hoadley KA, Akbani R, et al. Comprehensive molecular characterization of muscle-invasive bladder cancer. Cell 2018174 1033. (https://doi.org/10.1016/j. cell.2018.07.036)

10 Soukup V, Kalousova M, Capoun O, Sobotka R, Breyl Z, Pesl M, Zima T \& Hanus T. Panel of urinary diagnostic markers for noninvasive detection of primary and recurrent urothelial urinary bladder carcinoma. Urologia Internationalis 201595 56-64. (https:// doi.org/10.1159/000368166)

11 Dieplinger B, Gegenhuber A, Haltmayer M \& Mueller T. Evaluation of novel biomarkers for the diagnosis of acute destabilised heart failure in patients with shortness of breath. Heart 200995 1508-1513. (https://doi.org/10.1136/hrt.2009.170696)

12 Larsen AI, Helle KB, Christensen M, Kvaloy JT, Aarsland T \& Dickstein K. Effect of exercise training on chromogranin A and relationship to N-ANP and inflammatory cytokines in patients with chronic heart failure. International Journal of https://ec.bioscientifica.com https://doi.org/10.1530/EC-19-0068 (c) 2019 The authors Published by Bioscientifica Ltd

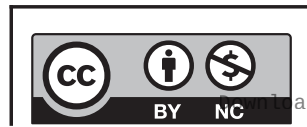

This work is licensed under a Creative Commons Attribution-NonCommercial 4.0 International License. ded from Bioscientifica.com at 04/26/2023 10:23:50AM 
Cardiology 2008127 117-120. (https://doi.org/10.1016/j. ijcard.2007.04.012)

13 Spadaro A, Ajello A, Morace C, Zirilli A, D'Arrigo G, Luigiano C, Martino F, Bene A, Migliorato D, Turiano S, et al. Serum chromogranin-A in hepatocellular carcinoma: diagnostic utility and limits. World Journal of Gastroenterology 200511 1987-1990. (https:// doi.org/10.3748/wjg.v11.i13.1987)

14 Tsao KC, Hong JH, Wu TL, Chang PY, Sun CF \& Wu JT. Elevation of CA 19-9 and chromogranin A, in addition to CA 125, are detectable in benign tumors in leiomyomas and endometriosis. Journal of Clinical Laboratory Analysis 200721 193-196. (https://doi. org/10.1002/jcla.20168)

15 Sciola V, Massironi S, Conte D, Caprioli F, Ferrero S, Ciafardini C, Peracchi M, Bardella MT \& Piodi L. Plasma chromogranin A in patients with inflammatory bowel disease. Inflammatory Bowel Diseases 200915 867-871. (https://doi.org/10.1002/ibd.20851)

16 Massironi S, Fraquelli M, Paggi S, Sangiovanni A, Conte D, Sciola V, Ciafardini C, Colombo M \& Peracchi M. Chromogranin A levels in chronic liver disease and hepatocellular carcinoma. Digestive and Liver Disease 200941 31-35. (https://doi.org/10.1016/j.dld.2008.05.002)

17 Ziegler MG, Kennedy B, Morrissey E \& O'Connor DT. Norepinephrine clearance, chromogranin A and dopamine beta hydroxylase in renal failure. Kidney International 199037 1357-1362. (https://doi.org/10.1038/ki.1990.122)

18 Kurnatowska I \& Nowicki M. Serum chromogranin A concentration and intradialytic hypotension in chronic haemodialysis patients. International Urology and Nephrology 200638 701-705. (https://doi. org/10.1007/s11255-005-0078-7)

19 Niedworok C, Tschirdewahn S, Reis H, Lehmann N, Szucs M, Nyirady P, Romics I, Rubben H \& Szarvas T. Serum chromogranin A as a complementary marker for the prediction of prostate cancerspecific survival. Pathology Oncology Research 201723 643-650. (https://doi.org/10.1007/s12253-016-0171-5)

20 Krabbe JG, Monaghan PJ, Russell J \& de Rijke YB. Analytical evaluation of a second generation assay for chromogranin A: a dual-site study. Clinical Chemistry and Laboratory Medicine 201654 e139-e142. (https://doi.org/10.1515/cclm-2015-0782)

21 Mathis G. Rare earth cryptates and homogeneous fluoroimmunoassays with human sera. Clinical Chemistry 199339 1953-1959.

22 Niedworok C, vom Dorp F, Tschirdewahn S, Rubben H, Reis H, Szucs M \& Szarvas T. Validation of the diagnostic and prognostic relevance of serum MMP-7 levels in renal cell cancer by using a novel automated fluorescent immunoassay method. International Urology and Nephrology 201648 355-361. (https://doi.org/10.1007/s11255015-1185-8)

23 Tschirdewahn S, Reis H, Niedworok C, Nyirady P, Szendroi A, Schmid KW, Shariat SF, Kramer G, vom Dorp F, Rubben H, et al. Prognostic effect of serum and tissue YKL-40 levels in bladder cancer. Urologic Oncology 201432 663-669. (https://doi.org/10.1016/j. urolonc.2014.02.004)

24 Ohl F, Jung M, Radonic A, Sachs M, Loening SA \& Jung K. Identification and validation of suitable endogenous reference genes for gene expression studies of human bladder cancer. Journal of Urology 2006175 1915-1920. (https://doi.org/10.1016/S00225347(05)00919-5)

25 Vandesompele J, De Preter K, Pattyn F, Poppe B, Van Roy N, De Paepe A \& Speleman F. Accurate normalization of real-time quantitative RT-PCR data by geometric averaging of multiple internal control genes. Genome Biology 20023 RESEARCH0034. (https://doi. org/10.1186/gb-2002-3-7-research0034)

26 Tropea F, Baldari S, Restifo G, Fiorillo MT, Surace P \& Herberg A Evaluation of chromogranin A expression in patients with nonneuroendocrine tumours. Clinical Drug Investigation 200626 715-722. (https://doi.org/10.2165/00044011-200626120-00005)

27 Giovanella L, Marelli M, Ceriani L, Giardina G, Garancini S \& Colombo L. Evaluation of chromogranin A expression in serum and tissues of breast cancer patients. International Journal of Biological Markers 200116 268-272. (https://doi. org/10.1177/172460080101600408)

28 Ronkainen H, Soini Y, Vaarala MH, Kauppila S \& Hirvikoski P. Evaluation of neuroendocrine markers in renal cell carcinoma. Diagnostic Pathology 20105 28. (https://doi.org/10.1186/ 1746-1596-5-28)

29 Stein JP \& Skinner DG. Radical cystectomy for invasive bladder cancer: long-term results of a standard procedure. World Journal of Urology 200624 296-304. (https://doi.org/10.1007/s00345-006-0061-7)

30 Rausch S, Hennenlotter J, Todenhofer T, Aufderklamm S, Schwentner C, Sievert KD, Stenzl A \& Gakis G. Impaired estimated glomerular filtration rate is a significant predictor for non-muscleinvasive bladder cancer recurrence and progression - introducing a novel prognostic model for bladder cancer recurrence. Urologic Oncology 201432 1178-1183. (https://doi.org/10.1016/j. urolonc.2014.05.009)

Received in final form 21 March 2019

Accepted 17 April 2019

Accepted Preprint published online 18 April 2019
This work is licensed under a Creative Commons Attribution-NonCommercial 4.0 International License. ded from Bioscientifica.com at 04/26/2023 10:23:50AM 